\title{
Oral Hygiene Maintenance Via Teledentistry in Orthodontic Patients: A Randomized Controlled Trial
}

Erum Behroz Khan ${ }^{1}$

Mairah Shah ${ }^{2}$

S.M. Tariq Rafi ${ }^{3}$

Sadia Rizwan ${ }^{4}$

Zuhair Ahmed

Samar Fatima ${ }^{6}$
BDS, FCPS, C-Orth, MTFPDP, C-HPE

BDS

MBBS, DLO, FCPS, FICS, FRCS

BDS, FCPS

BDS

BDS

OBJECTIVES: To determine the extent of improvement in oral hygiene via text message reminder in patients undergoing fixed orthodontic treatment.

METHODOLOGY: The participants were from 13 to 30 years of age recruited from Department of Orthodontics, Sindh Institute of Oral Health Sciences, Jinnah Sindh Medical University, Karachi, Pakistan. The sample comprised of 70 patients (59 female and 11 male) about to have fixed orthodontic treatment, 35 in text message group and 35 in control group. Patient's plaque index was evaluated at baseline and after 30 days.

RESULTS: Text message group had highly significant $(\mathrm{P} \leq .05)$ plaque index score (PostPI) than control group.

CONCLUSIONS: Orthodontic patients frequently reminded to brush have better oral hygiene.

KEYWORDS: Plaque index, Text message, Oral hygiene, Orthodontics.

HOW TO CITE: Khan EB, Shah M, Rafi S.M.T, Rizwan S, Ahmed Z, Fatima S. Oral hygiene maintenance via teledentistry in orthodontic patients: A randomized controlled trial. J Pak Dent Assoc 2021;30(1):34-38.

DOI: https://doi.org/10.25301/JPDA.301.34

Received: 20 August 2019, Accepted: 23 November 2020

\section{INTRODUCTION}

$\mathrm{M}$ outh cleanliness is paramount for the protection of oral health as it eliminates microbial plaque, preventing it from accumulating on teeth and gingivae. Saliva is a natural cleaning agent that removes supragingival plaque in the oral cavity. However, although such mechanisms might eradicate food debris, they do not adequately remove dental plaque. Therefore, regular personal oral hygiene is necessary to eliminate supragingival plaque. The most widely used way of actively removing plaque at home is brushing of the teeth. Research has proved that tooth

1. Assistant Professor, Supervisor FCPS-Training Program, Department of Orthodontics, Sindh Institute of Oral Health Sciences, Karachi, Sindh, Pakistan.

2. Fellowship of College of Physicians and Surgeons Part 2 Trainee, Department of, Sindh Institute of Oral Health Sciences, Karachi, Sindh, Pakistan.

3. Vice Chancellor, Jinnah Sindh Medical University, Karachi, Sindh, Pakistan.

4. Assistant Professor, Department of Orthodontics, Dr. Ishrat U1 Ibad Khan Institute of Oral Health Sciences, DUHS, Karachi, Sindh, Pakistan.

5. Fellowship of College of Physicians and Surgeons Part 2 Trainee, Department of Orthodontics, Sindh Institute of Oral health sciences, Karachi, Sindh, Pakistan.

6. Fellowship of College of Physicians and Surgeons Part 2 Trainee, Department of Orthodontics, Sindh Institute of Oral health sciences, Karachi, Sindh, Pakistan.

Corresponding author: "Dr. Mairah Shah" < myrashahsyed@gmail.com > brushing and other mechanical cleansing methods can reliably control plaque, if cleaning is sufficiently thorough and performed regularly. ${ }^{1}$ However, this can be challenging to the patients who have multi bracket fixed appliances that are required for the correction of orthodontic malocclusion. ${ }^{2}$ Microbial colonization of the dental and gingival tissues is a negative consequence of orthodontic treatment. Fixed orthodontic appliances inside the mouth causes qualitative and quantitative changes by altering the physicochemical state of bacterial growth. The multi bracket system encourages the development of dental plaque and hinders its elimination. ${ }^{3}$ Plaque formation in these patients is 2 to 3 times greater than levels in high plaque-forming adults without appliances. ${ }^{4}$ Numerous studies have shown oral hygiene declines after initial period of bonding in orthodontic patients as judged by plaque and gingival indices. Insufficient oral hygiene before or throughout orthodontic treatment can also lead to white spot lesions. In another study, it was demonstrated that unwanted side effects appeared at the end of the orthodontic treatment due to deteriorating oral hygiene, that led to disappointing results and early removal of appliance. ${ }^{5}$ A major side effect of fixed orthodontic treatment is 
appearance of caries development on the facial surface in the form of white spot lesions. Studies have shown WSL after fixed appliance therapy in $50 \%$ to $70 \%$ of patients. ${ }^{6}$ It has been proved that amount of plaque formation in orthodontic patients can be reduced by reminder therapy. ${ }^{7}$ "Tele" is a word derived from Greek meaning distance, and "mederi" is a word derived from Latin meaning "to heal". Tele-medicine delivers healthcare across large geographic distances through information based technologies and communication system. ${ }^{8}$ Likewise communicating with orthodontic patients through the medium of information technology instead of face to face to take care of their appliance, to guide and treat them from a distance is known as Tele- orthodontics. ${ }^{9}$ Recent developments in technology like computers and smartphones have made management of orthodontic patients by tele-dentistry more feasible..$^{10}$ It has been shown that communicating with these patients through active reminders, increases their attendance on appointments, makes them take their medications on time and brings positive changes in their behavior. ${ }^{11}$

Patient's negligence in oral health during orthodontic treatment results in plaque accumulation that can lead to undesired side effects. Patients should be frequently reminded of its importance through oral hygiene instruction's text message. Our purpose to conduct this study is to determine the extent of improvement in oral hygiene in orthodontic patients by reminding via text message.

\section{METHODOLOGY}

This study was conducted as a randomized clinical trial, performed on 70 selected orthodontic patients, 35 in text message group and 35 in control group. The study was explained to patients or their parents and they were asked to sign informed consent forms. The sample comprised of 59 females and 11 male participants. They were from 13 to 30 years of age without any significant medical/ dental history recruited from Department of Orthodontics, Sindh Institute of Oral Health Sciences, Jinnah Sindh Medical University, Karachi, Pakistan. Duration of this study was 30 days. Ethical Approval was obtained (JSMU/IRB/2020/-312)

Inclusion criteria: Both genders were included i.e. male and female with the age range from 13 to 30 years. Patient's presenting with complain of mal aligned teeth, about to have fixed orthodontic treatment, internally motivated and having good oral health. Patients with crowding in different types of malocclusions (class I, II \& III). All these patients had smartphones and were able to read and write.

Exclusion criteria: Patient's with poor oral hygiene, patient's already on fixed orthodontic treatment, diabetic patients, betel nut chewers were excluded from the study.
All the participants received standard scaling and polishing 3 to 4 weeks prior to the treatment and their periodontal health was maintained till commencement of the treatment. These participants were then divided into two groups through sealed envelope technique i.e. principal investigator was given randomly generated treatment allocations within sealed opaque envelopes. Once a patient had consented to enter a trial an envelope was opened and the patient was then offered the allocated treatment regimen.

The control group was composed of 31 female and 4 male patients who were about to have fixed orthodontic treatment. Their plaque index was evaluated before fixed orthodontic treatment (PrePI), after the bonding they were given standardized oral hygiene instructions that included proper brushing and use of mouth wash. After 30 days their plaque index (PostPI) was reevaluated.

Text message group was composed of 28 female and 7 male patients who were about to have fixed orthodontic treatment. Their plaque index was evaluated before fixed orthodontic treatment, after bonding they were given standardized oral hygiene instruction in a similar manner as the control group. They received a text message reminder once each week for 30 days. The text message stated "Keep your smile shining, keep your teeth and braces clean, don't forget to Brush them for 2 minutes at least after every meal. See you soon." After 30 days their plaque index was reevaluated and their results were compared and contrasted with the control group.

The plaque index was scored as described by SilnessLöe plaque index. Silness-Löe was chosen over other plaque indexes, as it assesses thickness of plaque at gingival margins rather than on its coronal extent on the tooth surface area. It is calculated on the basis of recording soft and hardened plaque deposits on following teeth: 16, 12, 24, 44, 32, 36. The buccal, lingual, mesial and distal surfaces (Four surfaces) of the each tooth were given a score between 0-3 as described in table 1. In order to calculate each tooth's plaque index, the scores for its four surfaces were added and divided by

Table 1: Silness- Löe plaque index

\begin{tabular}{|l|l|}
\hline 0 & No plaque \\
\hline 1 & $\begin{array}{l}\text { A film of plaque adhering to the free gingival margin and adjacent area of } \\
\text { the tooth. The plaque may be seen in situ only after application of } \\
\text { disclosing solution or by using the probe on the tooth surface. }\end{array}$ \\
\hline 2 & $\begin{array}{l}\text { Moderate accumulation of soft deposits within the gingival pocket, or the } \\
\text { tooth and gingival margin which can be seen with the naked eye }\end{array}$ \\
\hline 3 & $\begin{array}{l}\text { Abundance of soft matter within the gingival pocket and/or on the tooth and } \\
\text { gingival margin. }\end{array}$ \\
\hline
\end{tabular}

four. Then all the six teeth mentioned above were summed and divided by six to calculate the index for the patient. This score was graded as described in table 2 . The collected data 
Table 2: Plaque index grading

\begin{tabular}{|l|l|}
\hline 0 & Excellent \\
\hline $0.1-0.9$ & Good \\
\hline $1.0-1.9$ & Fair \\
\hline $2.0-3.0$ & Poor \\
\hline
\end{tabular}

was analyzed through SPSS 22, t-test was applied to compare scores of text message group with control group, keeping $\mathrm{P}$ value $\leq .05$.

\section{RESULTS}

All the data was analyzed using SPSS 22, the difference between the means of two groups was found by applying independent $t$ - test. The test results were significant $(\mathrm{P} \leq .05)$ for post treatment plaque index of text message group in comparison to control group. Both groups had almost similar pretreatment plaque index, $0.67 \pm 0.17$ in control group and $0.56 \pm 0.11$ in text message group. There was difference in plaque index after 30 days, mean plaque index in control group was $1.18 \pm 0.31$ and in text message group was $0.68 \pm 0.13$ as shown in table 3 . Comparison of plaque index of these two groups showed significant difference after 30 days, as discussed in table 4.

Table 3: Plaque index mean and standard deviation

\begin{tabular}{|l|c|c|}
\hline \multicolumn{1}{|c|}{ Time } & Text Message Group & Control Group \\
\hline Baseline(PrePI) & $0.56 \pm 0.11$ & $0.67 \pm 0.17$ \\
\hline $\begin{array}{l}\text { After } 30 \\
\text { days(PostPI) }\end{array}$ & $0.68 \pm 0.13$ & $1.18 \pm 0.31$ \\
\hline
\end{tabular}

Table 4: T- Test Significant results after 30 days

\begin{tabular}{|c|c|c|c|c|}
\hline & \multicolumn{3}{|c|}{ t-test for Equality of Means } \\
\hline & & df & $\begin{array}{l}\text { Sig. (2- } \\
\text { tailed) }\end{array}$ & $\begin{array}{c}\text { Mean } \\
\text { Difference }\end{array}$ \\
\hline \multirow{2}{*}{$\begin{array}{l}\text { Plaque index score After } \\
\text { treatment }\end{array}$} & $\begin{array}{l}\text { Equal variances } \\
\text { assumed }\end{array}$ & 68 & .000 & -.49943 \\
\hline & $\begin{array}{l}\text { Equal variances not } \\
\text { assumed }\end{array}$ & 45.945 & .000 & -.49943 \\
\hline
\end{tabular}

\section{DISCUSSION}

The principal etiologic factor behind the formation of periodontal disease and dental caries, is a very complicatedly organized form of biofilm known as dental plaque. Orthodontic treatment with fixed appliances results in plaque accumulation. It Is essential to evaluate of dental plaque regularly to maintain the cleanliness in oral cavity of individual patients who are going through fixed appliance treatment. ${ }^{12}$

Good communication with patients can be maintained through text messaging. This will make the patient believe about how much the orthodontist is involved in the patient's treatment and how much he or she is concerned regarding health of the patient between long gaped appointments. This has been proved to be very influential in satisfying patients and encouraging a good relationship between orthodontist and patient. The influence of word of mouth, or in this case the written word, seems to be extremely useful when it serves as a medium of communication between orthodontist and their young patients. ${ }^{13}$ The current study was designed as a randomized clinical trial of 70 orthodontic patients, about to have fixed orthodontic appliance treatment. This study was carried out to check plaque index in orthodontic patients who are reminded oral hygiene instructions through text message and to compare their plaque index score with control/ conventional group. Both groups i.e. control group and text message group participated in this study for 30 days. Both groups had similar plaque index scores $(95.7 \%$ rated as good) before treatment. After 30 days of treatment there

Table 5: Treatment Plaque Index Grading

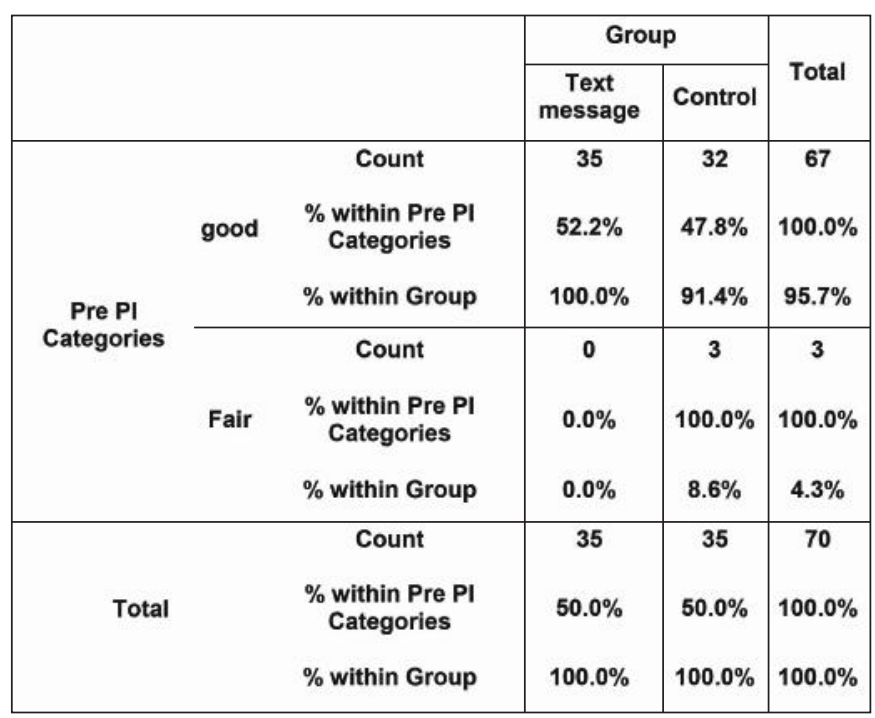

wasn't much difference in text message group's PI (97.1\% rated as good, $2.9 \%$ rated as fair) but there was a notable difference in control group's PI (20\% rated as good, $77.1 \%$ rated as fair and $2.9 \%$ as poor) as described in table 5 . Comparison between the two groups shows significant difference in plaque index after 30 days of treatment with $\mathrm{P}$ value $\leq .05$. Limitations of the study were the small sample size and patient compliance in data collection.

In a similar study carried out in Genova, it was 
demonstrated that participants receiving post-treatment reassurance through communication(text message and calls) reported more compliance in oral hygiene than the control group patients. ${ }^{14}$ In 2017, in Italy a app-based approach was applied to standard maintenance of oral hygiene to improve oral health and hygiene compliance in a teenage patients group who had fixed multi bracket appliances. ${ }^{15}$ Although these methods are effective in decreasing plaque accumulation during treatment, however, text messaging is more convenient. It is has been proved that around $75 \%$ of smartphone users between the age range of 12-17 years often communicate through text messages. ${ }^{4}$ In another study conducted in Pakistan, showed decreased modified gingival index, plaque index and bleeding index after 30-60 days of orthodontic treatment in text message group and proved it successful in improving oral hygiene. ${ }^{5}$ Many studies have demonstrated that fixed orthodontic treatment's initial months are very demanding for patients as they have to get acquainted with new manual skills for maintenance of orthodontic appliance in an appropriate hygienic condition. ${ }^{15}$ Our study's aim is to evaluate changes in oral hygiene after one month in both groups (text message and control) on a larger population and to familiarize them to oral hygiene measures for fixed orthodontic appliance. Limitations of the study were the small sample size, limited funding and time constraint.

Table 6: Post Treatment Plaque Index Grading

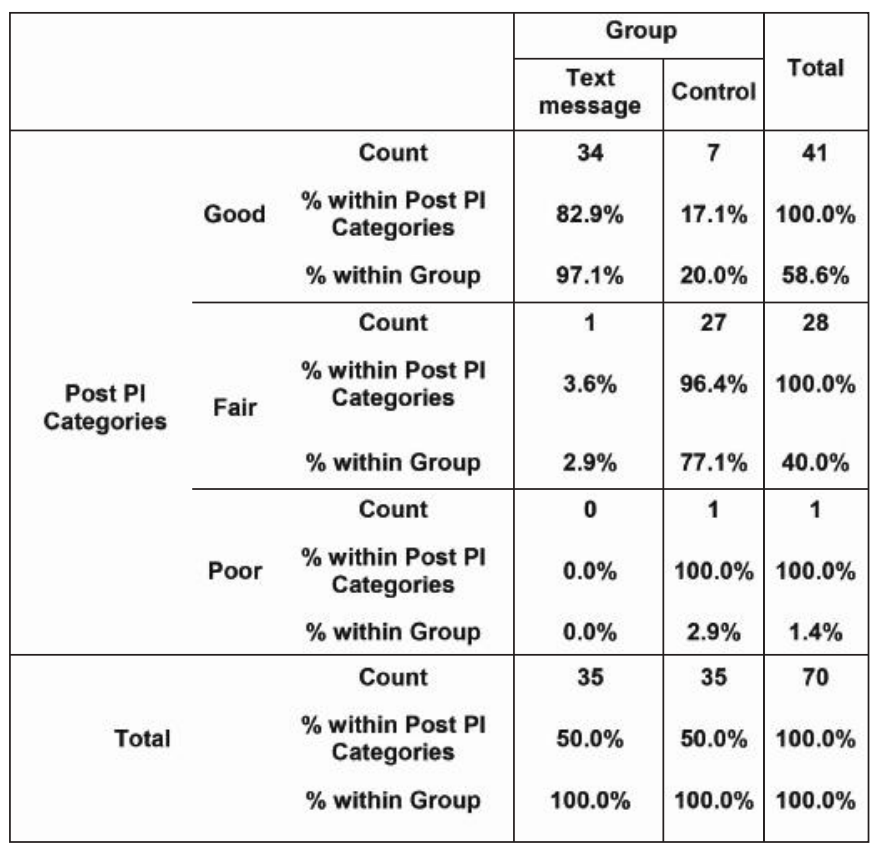

\section{CONCLUSION}

- Orthodontic patients receiving text message reminders have better oral hygiene than conventional group
- Patients should be frequently reminded to brush and maintain their oral hygiene.

\section{CONFLICT OF INTEREST}

None declared

\section{REFERENCES}

1. Van Der Weijden F, Slot DE. Oral hygiene in the prevention of periodontal diseases: the evidence. J Periodontol 2000. 2011;55: 104-23.

https://doi.org/10.1111/j.1600-0757.2009.00337.x

2. Islam ZU, Shaikh A, Fida M. Plaque index in multi-bracket fixed appliances. J Colle Physic Surg-Pak: JCPSP. 2014;24:791-95.

https://ecommons.aku.edu/pakistan_fhs_mc_surg_orthop/63/

3. Ousehal L, Lazrak L, Es-said R, Hamdoune H, Elquars F, Khadija A. Evaluation of dental plaque control in patients wearing fixed orthodontic appliances: a clinical study. Int Orthod. 2011;9:140-55. https://doi.org/10.1016/j.ortho.2010.12.013

4. Klukowska M, Bader A, Erbe C, Bellamy P, White DJ, Anastasia MK, et al. Plaque levels of patients with fixed orthodontic appliances measured by digital plaque image analysis. Am J Orthod Dentofacial Orthop. 2011;139:e463-e70.

https://doi.org/10.1016/j.ajodo.2010.05.019

5. Iqbal J, Awan R, Parvez MA, ul Haq A, Gardezi AA, Irfan S. Effectiveness of text message instructions on oral hygiene for orthodontic patients. Pak Oral Dent J. 2017;37:278-82. http://www.podj.com.pk/index.php/podj/article/view/97

6. Øgaard B, Larsson E, Henriksson T, Birkhed D, Bishara SE. Effects of combined application of antimicrobial and fluoride varnishes in orthodontic patients. Am J Orthod Dentofacial Orthop. 2001;120: 28-35.

https://doi.org/10.1067/mod.2001.114644

7. Lima IFP, de Andrade Vieira W, de Macedo Bernardino Í, Costa PA, Lima APB, Pithon MM, et al. Influence of reminder therapy for controlling bacterial plaque in patients undergoing orthodontic treatment: A systematic review and meta-analysis. Angle Orthod. 2018;88:48393.

https://doi.org/10.2319/111117-770.1

8. Batham PR, Kalia UDP, Dilliwal S. Teledentistry and its role in orthodontic patient management. Int J Stomatolo Occlusion Med. 2014;7:6-12.

https://doi.org/10.1007/s12548-013-0097-4

9. Kravitz N, Burris B, Butler D, Dabney C. Teledentistry, Do-ItYourself Orthodontics, and Remote Treatment Monitoring. J Clin orthodo. 2016;50:718.

10. Costa ALPd, Silva AA, Pereira CB. Tele-orthodontics: tool aid to clinical practice and continuing education. Dental Press J Orthod. 
Khan EB/ Shah M/ Rafi S.M.T/

Rizwan S/ Ahmed Z/ Fatima S

2011;16:15-21.

https://doi.org/10.1590/S2176-94512011000600003

11. Eppright M, Shroff B, Best AM, Barcoma E, Lindauer SJ. Influence of active reminders on oral hygiene compliance in orthodontic patients. Angle Orthod. 2013;84:208-13.

https://doi.org/10.2319/062813-481.1

12. Al-Anezi SA, Harradine NW. Quantifying plaque during orthodontic treatment: a systematic review. Angle Orthod. 2012;82:748-53. https://doi.org/10.2319/050111-312.1

13. Brent Bowen T, Rinchuse DJ, Zullo T, DeMaria ME. The influence of text messaging on oral hygiene effectiveness. Angle Orthod.
Oral hygiene maintenance via teledentistry in orthodontic patients

2015;85:543-48.

https://doi.org/10.2319/071514-495.1

14. Cozzani M, Ragazzini G, Delucchi A, Mutinelli S, Barreca C, Rinchuse DJ, et al. Oral hygiene compliance in orthodontic patients: a randomized controlled study on the effects of a post-treatment communication. Prog Orthod. 2016;17:41.

https://doi.org/10.1186/s40510-016-0154-9

15. Zotti F, Dalessandri D, Salgarello S, Piancino M, Bonetti S, Visconti $\mathrm{L}$, et al. Usefulness of an app in improving oral hygiene compliance in adolescent orthodontic patients. Angle Orthod. 2016;86:101-07. https://doi.org/10.2319/010915-19.1 